\title{
Situación laboral de las mujeres inmigrantes en España*
}

\author{
Laura MOLPECERES ÁLVAREZ \\ Técnica de ENRED Consultoría \\ lauram@enred.es
}

Recibido: 04-07-11

Aceptado: 26-09-11

\section{RESUMEN}

El artículo presenta los resultados obtenidos sobre la situación laboral de las mujeres extranjeras en España a partir de la explotación de diversas fuentes estadísticas secundarias y especialmente de los microdatos de la EPA. Se realiza un análisis descriptivo de dichas fuentes, complementado con un análisis de correspondencias múltiples que presenta las variables más significativas del espacio socioeconómico de estas mujeres. Ambas aproximaciones sirven para mostrar la diversidad de situaciones laborales que se dan entre las inmigrantes. Al mismo tiempo, los resultados desvelan que el país de procedencia es la variable que guarda una mayor relación con respecto al tipo de empleo al que acceden las mujeres inmigrantes.

Palabras clave: Género, mujeres inmigrantes, empleo, España.

\section{The employment status of immigrants women in Spain}

\begin{abstract}
The article presents an analysis of the employment situation of foreign women in Spain based on the analysis of different secondary data sources and mainly EPA microdata. A descriptive analysis of these sources is performed, complemented by a multiple correspondence analysis on the most significant variables of migrant women's socioeconomic space. Both approaches are used to bring out the diversity of work situations that involves migrant women. At the same time, it reveals how the country of origin is the variable more related with the kind of employment.
\end{abstract}

Keywords: Gender, migrant workers, employment, Spain.

REFERENCIA NORMALIZADA

Molpeceres Álvarez, L. (2012). Situación laboral de las mujeres inmigrantes en España. Cuadernos de Relaciones Laborales Vol. 30, núm. 1, p. 91-113

* Marta Óngil López y Ma Ángeles Sallé Alonso (ENRED CONSULTORÍA) han colaborado en la elaboración del artículo. 
SUMARIO: Introducción. 1. Migraciones, género y empleo. 2. Situación laboral de las mujeres inmigrantes en España. 3. Diversidad y matices entre las trabajadoras extranjeras. 4. La procedencia, variable ilustrativa. 5. Conclusiones. Bibliografía

\section{Introducción}

El artículo presenta los resultados obtenidos sobre la situación laboral de las mujeres extranjeras en España a partir de la explotación de fuentes estadísticas secundarias, principalmente los microdatos de la EPA. Dicha explotación se ha realizado en el marco del estudio "Análisis de la situación laboral de las mujeres inmigrantes: modalidades de inserción, sectores de ocupación e iniciativas empresariales" (Sallé, Molpeceres y Ongil, 2009), financiado por el Instituto de la Mujer. Se ofrece un análisis descriptivo de dichas fuentes, complementado con un análisis de correspondencias múltiples sobre las variables más significativas del espacio socioeconómico de estas mujeres. Ambas aproximaciones sirven para mostrar los matices y la diversidad de situaciones laborales que se dan entre las inmigrantes. Al mismo tiempo desvela cómo la variable país de procedencia es la que guarda una mayor relación respecto a su posición en el empleo.

En primer lugar se procede a contextualizar los movimientos migratorios internacionales y, más en concreto, el fenómeno de la "feminización de las migraciones" y las dinámicas del mercado laboral. Seguidamente se repasan los rasgos característicos de la situación laboral de las mujeres extranjeras en España, poniendo el foco de atención en la concentración sectorial y ocupacional y las condiciones laborales precarias. Sin embargo, la situación laboral de estas mujeres presenta rasgos diferenciales que son interesantes analizar y que el artículo muestra sobre la base de los resultados obtenidos en el estudio antes mencionado, tomando la nacionalidad como uno de los principales predictores de la situación de empleo. El texto concluye con un apartado de conclusiones.

\section{Migraciones, género y empleo}

La investigación en torno a los movimientos migratorios es muy extensa y ha sido abordada desde diversas disciplinas (economía, antropología, sociología,...). El interés sobre este fenómeno se ha intensificado en las últimas décadas, motivado, en gran medida, por el crecimiento que han experimentado las migraciones internacionales. Según estimaciones de la ONU, desde la década de los 60, el número de migraciones internacionales se ha duplicado, llegando a alcanzar una cifra de 190 millones de personas, el 3\% de la población mundial (United Nations, 2009).

Las mujeres representan más de la mitad en los movimientos migratorios procedentes de Europa, Oceanía y de la antigua Unión Soviética; en torno al 50\% para América Latina, Caribe y América del Norte; y son minoría en los desplazamientos de África y Asia (Morrison, Schiff y Sjöblom, 2007). Esta cuestión, es decir, el peso y la cualidad de la participación de las mujeres en los flujos migratorios, no sería 
relevante si, como en el resto de dimensiones sociales, tales flujos no estuvieran influenciados y explicados por las relaciones de género.

Las relaciones de género afectan a la hora de explicar quiénes y por qué razones emigran, cómo se toma la decisión de hacerlo, así como las consecuencias sobre las propias personas migrantes y los impactos tanto en las comunidades de origen y de destino (Jolly y Reeves, 2005). El género, introducido en los modelos empíricos de investigación, se convierte de este modo en una variable relevante a la hora de explicar las formas de la migración y sus resultados (Morrison, Schiff y Sjöblom, 2007).

Sin embargo, el esfuerzo e interés en la incorporación de la perspectiva de género dentro de la investigación sobre migraciones es más bien reciente (González, 2008). Hasta hace relativamente poco, las teorías y modelos migratorios no daban cuenta del papel y la participación de las mujeres, o bien aparecían reflejadas como meras acompañantes de sus homólogos masculinos (Camacho, 2010). Habrá que esperar a la denominada "feminización de las migraciones", caracterizada por un aumento del volumen de mujeres iniciadoras de la cadena migratoria en algunos flujos (Martínez, 2007; Orozco, Paiewonsky y García, 2008), para que comience a visibilizarse y ponerse en valor su participación.

En términos laborales, este crecimiento de los flujos migratorios y el protagonismo de las mujeres asumido en ellos, se inscribe en un contexto global de dualización del mercado laboral en las "naciones centrales" a partir de la generación de sectores de empleo secundarios (Cachón, 2004). La demanda de empleo en determinados sectores que la población autóctona no cubre se convierte, de este modo, en nicho de ocupación para personas extranjeras procedentes de países con inferiores o escasas oportunidades laborales.

$\mathrm{Si}$ a ello añadimos la componente de género -que se refleja, entre otras cosas, en la segregación sectorial y ocupacional por sexos del mercado laboral ${ }^{1}$ - el resultado es que, además, dentro de esos nichos de empleo las mujeres terminan por ocupar sectores "feminizados".

Un peso singular de la concentración sectorial del empleo de estas mujeres lo acapara, precisamente, el sector del cuidado y el servicio doméstico. Y es que el protagonismo de las mujeres dentro de los flujos migratorios se contextualiza, también, en una dinámica global de transferencia de cargas reproductivas desde los países más desarrollados a los menos desarrollados, en un momento de incorpora-

${ }^{1}$ Una segregación ocupacional por sexos que, lejos de disminuir, se ha ido acentuando con el paso de los años (Maté, Nava y Rodríguez, 2002). Por su parte, la segregación sectorial se habría reducido en los últimos años, pero muy ligeramente (Cáceres, Escot, Fernández y Saiz, 2004). De esa forma "frente a lo que podría esperarse, la segregación ocupacional no se ha visto reducida con la mayor participación laboral de la mujer; al contrario, presenta cierta tendencia al alza desde finales de los años ochenta" (Ibáñez, 2008; 89). 
ción masiva de las mujeres al trabajo productivo y de necesidad de cubrir las tareas domésticas y de cuidado, que salen al mercado y son satisfechas, en las "naciones centrales", por mujeres procedentes de otros países.

La migración internacional de las mujeres revela, así, una emergente internacionalización del trabajo reproductivo (Parella, 2003) y el surgimiento de las cadenas globales de cuidados, uno de los fenómenos más paradigmáticos del actual proceso de feminización de las migraciones en el contexto de la globalización (Orozco, 2007).

La inserción y situación laboral de las mujeres migrantes, dado el volumen y la relevancia que han adquirido en los movimientos migratorios, con características propias y diferenciales respecto a la migración masculina, se ha convertido de este modo en un tema de interés recurrente para la investigación social.

\section{Situación laboral de las mujeres inmigrantes en España}

En el caso concreto de España, los análisis en este ámbito se han intensificado a partir de los últimos años de la década de los noventa, debido a que las dinámicas migratorias comentadas se han producido de un modo mucho más intenso a partir de ese periodo. España ha experimentado, de hecho, un crecimiento súbito y acelerado de su población inmigrante. La demanda de empleo, provocada por el crecimiento económico de los últimos años, ha ocasionado que la llegada de inmigrantes económicos a España se haya producido a lo largo de un periodo de tiempo inferior al que han experimentado otros países de su entorno receptores de flujos migratorios, habiéndose multiplicado por seis la población de personas extranjeras en tan solo una década, tanto de hombres como de mujeres (INE, 2010).

La inserción y situación laboral de las mujeres inmigrantes ha pasado, de este modo, a ocupar un considerable espacio de la investigación social, a la vez que se ha convertido en un tema de preocupación para las instituciones (Colectivo Ioé, 1998). Así, el repaso de la literatura nos habla de unas características generalizadas y comunes respecto a la situación laboral de las mujeres inmigrantes en España, en las que la concentración sectorial y ocupacional, la precariedad en las condiciones laborales o la escasa movilidad suelen ser reiterativas.

La bibliografía especializada pone en evidencia, en primer lugar, la elevada concentración sectorial y ocupacional de las trabajadoras extranjeras en nichos de empleo de menor cualificación, más intensa que la que se da entre los hombres extranjeros y entre las mujeres españolas. Y es que casi la mitad de las mujeres extranjeras ocupadas desarrollan trabajos no cualificados y en sectores como la hostelería, el comercio o el trabajo doméstico (INE, 2011). Esta tendencia se observa también en otros países; sin embargo, para el caso de España, reviste particularidades propias. De hecho, la inserción laboral de las inmigrantes no comunitarias sólo se comprende en el contexto de la estructura ocupacional española, caracterizada por la terciarización, precarización y segmentación del empleo (Colectivo Ioé, 1998). 
La concentración en empleos poco cualificados trae aparejado otro rasgo típico de la situación laboral de estas mujeres, la sobrecualificación o subempleo, si se atiende al nivel de cualificación desempeñado en el empleo en relación con su nivel educativo $^{2}$. A la concentración sectorial en ocupaciones y sectores descualificados van asociadas, también, condiciones de trabajo precarias. Les son propias a las mujeres extranjeras, por ejemplo, unas retribuciones bastante inferiores que las que perciben el resto de grupos, una mayor temporalidad, jornadas y horarios poco compatibles con otras facetas de la vida y multiempleo, por no hablar de las situaciones de irregularidad laboral que padecen muchas de ellas (Sallé, Molpeceres y Ongil, 2009).

La concentración sectorial y ocupacional para aquellas mujeres que ocupan empleos precarios y de menor cualificación, además, no parece mejorar con un mayor tiempo de residencia en España. Nueve de cada diez mujeres con más de tres años de residencia en España que comenzaron a trabajar en el sector doméstico continúan en él, mientras que ocho de cada diez que inician su trayectoria en trabajos no cualificados permanecen en la misma situación (ENI, 2007). Frente a esta circunstancia, diversas investigaciones señalan que el empleo por cuenta propia constituye la única estrategia a su alcance para lograr la movilidad laboral (Solé, Parella y Cavalcanti, 2007). La "empresarialidad étnica" se considera, por tanto, una estrategia de movilidad laboral (Oso, 2004). Sin embargo, las tasas de salarización entre las mujeres extranjeras son muy elevadas, llegando a alcanzar el 93\% (INE, 2011).

A este panorama de acentuada precariedad se unen, por último, unas altas tasas de desempleo que, hasta la reciente crisis económica, se habían situado por encima de las del resto de grupos considerados (hombres y mujeres españolas, hombres extranjeros) pero que en el actual contexto laboral presentan unos índices por debajo del de sus homólogos masculinos, con una tasa de paro del $27 \%$ entre las mujeres extranjeras frente al 33\% de los hombres extranjeros (INE, 2010).

La dimensión sectorial de la crisis económica, que afectó en un principio a sectores con una alta participación de trabajadores extranjeros varones (caso de la construcción, por ejemplo) es una de las razones que están detrás de la distancia favorable a las mujeres extranjeras. Para algunos autores, la segregación laboral de las mujeres, en general, y de las extranjeras, en particular, suponen nichos de empleo en los que se evita la competencia con los hombres, de forma que en momentos de recesión, como el actual, proporcionan cierta protección contra el desempleo (Ibáñez, 2008). De hecho, aunque el crecimiento de las tasas de desempleo ha sido muy elevado en todos los grupos poblaciones, este incremento ha sido inferior en el caso de las mujeres que en el de los hombres, y menos elevado en el caso de las mujeres extranjeras (INE, 2011).

\footnotetext{
${ }^{2}$ Más de la mitad de las mujeres extranjeras en España poseen un nivel educativo de secundaria y un 20\% Educación Superior (EPA, INE. IIT2010).
} 
Actualmente, con un crecimiento del desempleo focalizado en el sector servicios, la tendencia puede revertirse y habrá que seguir de cerca los resultados sobre el desempleo "femenino".

A modo de síntesis, el panorama presentado apunta, de esta manera, a cómo las particularidades del mercado laboral español y, por ende, de su economía y estructura ocupacional, se apoyan en la interacción de dimensiones de clase, etnia y género que abocan a las mujeres inmigrantes a una participación laboral máximamente precaria y marginal. El vínculo entre mujer inmigrante y ocupaciones "precarias" se va consolidando a través de los prejuicios, los estereotipos, el etnocentrismo y el androcentrismo de manera que:

el estatus de las mujeres inmigrantes se ve afectado por las restricciones de una estructura ocupacional sexualmente segregada, en la que las mujeres obtienen más bajos salarios, menor estabilidad y menos oportunidades de promoción que sus homólogos masculinos, independientemente de su capacitación. Sin embargo, los inconvenientes que entraña el hecho de ser inmigrante implica que la trabajadora inmigrante, en comparación con la mujer autóctona, se halle en los estratos más bajos de la estructura ocupacional, cubriendo los huecos laborales peor pagados, con menos prestigio social y eludibles por las trabajadoras autóctonas por ser emblemáticos de la discriminación de género (Parella, 2003; 139).

\section{Diversidad y matices entre las trabajadoras extranjeras}

Esta panorámica general responde a una situación de conjunto de las mujeres extranjeras en España que ofrece una visión sobre su estatus laboral y sus patrones de incorporación al mercado de trabajo. Sin embargo, no permite ahondar en las especificidades que están detrás de la diversidad de mujeres que llegan a nuestro país.

Partimos de la hipótesis de que esas características generales se dan principalmente sobre unos perfiles determinados de mujeres inmigrantes. No hay que olvidar que en España existe un importante volumen de población extranjera procedente de Reino Unido, Alemania o Argentina ${ }^{3}$, con una situación laboral diferenciada, aunque solamente se considere en términos de 'inactividad'. También hay que tener en cuenta que dentro de la inmigración de mujeres en España, son variados los proyectos, las trayectorias y los condicionantes de las mujeres que los protagonizan, por lo que dentro de estas características generales entendemos que existen particularidades o matices interesantes de analizar y mostrar.

\footnotetext{
${ }^{3}$ Por grupos de procedencia el mayor número de mujeres extranjeras en España está representado por rumanas (14\% de la población extranjera de mujeres), marroquíes $(9 \%)$ y ecuatorianas (7\%). Tras ellas se sitúa Reino Unido (7\%), Colombia (6\%), Bolivia (4\%), Argentina (3\%) y Alemania (3\%) (INE, 2010).
} 
Bajo estos supuestos, en el estudio editado por el Instituto de la Mujer "Análisis de la situación laboral de las mujeres inmigrantes: modalidades de inserción, sectores de ocupación e iniciativas empresariales" (Sallé, Molpeceres y Ongil, 2009) realizamos una explotación estadística descriptiva de fuentes secundarias, que nos permite ofrecer una visión más detallada de la diversificada realidad de la situación laboral de las mujeres inmigrantes en España.

Aunque las fuentes de base son la EPA, la Encuesta Nacional de Inmigrantes $(\mathrm{ENI})^{4}$ y los registros de afiliación a la Seguridad Social, también se ha recurrido a otras estadísticas como son la Encuesta de Migraciones, la Encuesta de Estructura Salarial o a los contratos registrados en el INEM.

A partir de este amplio análisis descriptivo pudimos constatar que la realidad laboral de las mujeres extranjeras en España ofrece diferencias, en las que la variable procedencia o nacionalidad aporta elementos diferenciadores significativos.

En primer lugar, la situación laboral de las 'inmigrantes económicas' del Este de Europa reviste características propias en comparación con las mujeres latinoamericanas, quienes se distancian a su vez de las procedentes de países africanos o de las del continente asiático. Sin contar con otras procedencias con un peso importante en España, como son las inmigrantes de Reino Unido, Alemania o Argentina.

Así, para las mujeres procedentes del Este europeo (mayoritariamente de Rumania) cabría resaltar su grado de sobrecualificación y su menor concentración "relativa" en el empleo doméstico que para otras procedencias, con una presencia algo más significativa en otras ramas como el comercio, la hostelería o las actividades inmobiliarias y servicios empresariales ${ }^{5}$. Presentan, asimismo, una movilidad laboral media entre sectores y ocupaciones, pero siempre en niveles de menor rango.

\footnotetext{
${ }^{4}$ La Encuesta Nacional de Inmigrantes (ENI), realizada en 2007, constituye una de las aproximaciones más relevantes de acercamiento al fenómeno de la inmigración desde el punto de vista estadístico en España. Representa, en este sentido, la primera Encuesta a nivel estatal avalada por el INE que se centra particularmente en el análisis de los itinerarios seguidos por las personas inmigrantes así como en determinados aspectos de su experiencia migratoria: decisión de emigrar, condiciones de llegada, situación familiar, trayectorias laborales y residenciales, relaciones con países de origen, estrategias de futuro, etc. La ENI ha posibilitado, de este modo, la unificación de muchas de las dimensiones a considerar en los procesos migratorios ofrecidos de manera dispersa por otras fuentes estadísticas del INE (Censo de Población y Viviendas, Movimiento Natural de la Población, Padrón Municipal, Encuesta de Población Activa, etc.).

${ }^{5}$ En esta categoría se incluyen una amplia diversidad de actividades (actividades jurídicas y de contabilidad, servicios de arquitectura e ingeniería, ensayos y análisis clínicos, publicidad, selección y colocación de personal, servicios de investigación y seguridad y otras actividades empresariales diversas) algunas de las cuales se intuye emplean en una importante proporción a mujeres extranjeras: actividades industriales y de limpieza, actividades de envasado y empaquetado o actividades de centro de llamadas. La explotación de
} 
Las mujeres de América Central y del Sur, pero fundamentalmente de la región andina (Ecuador, Colombia y Bolivia), son las que presentan las tasas de actividad y empleo más elevadas. Les es característico un nivel educativo de Secundaria, aunque con una proporción importante de estudios de Primaria y Superiores, por lo que en función de su distribución sectorial y ocupacional podría hablarse de cierto grado de sobrecualificación, aunque inferior al de las del Este europeo. Aparecen, así, para estas nacionalidades, elevados niveles de concentración sectorial y posiciones muy marcadas en los niveles más bajos de la escala laboral. Todas ellas muestran altos grados de concentración en las ramas de actividades de los hogares y las actividades inmobiliarias y de servicios empresariales. Las mujeres de Ecuador, además, se concentran en el comercio y la hostelería, las procedentes de Colombia en el comercio y las originarias de Bolivia en la hostelería. En torno al $80 \%$ de este grupo se emplean en trabajos no cualificados o en "servicios de restauración, personales, de protección o vendedoras de comercio", un porcentaje que se eleva a más del $90 \%$ entre las bolivianas (INE, 2007). Se identifica movilidad en sus trayectorias laborales, de carácter sectorial para las ecuatorianas y colombianas, y ocupacional para ecuatorianas y bolivianas, aunque es mucho más escasa para las que desempeñan trabajos no cualificados y empleos en el sector doméstico.

Dentro del grupo de las latinoamericanas, otra nacionalidad con un peso importante en España es la argentina, si bien se trata de mujeres que presentan una situación laboral distinta a la de las mujeres de regiones andinas. Su posición en niveles medios-altos en la escala laboral (técnicas y profesionales, personal administrativo), así como su más reducida presencia en el sector doméstico, las sitúa en otro perfil laboral. Se emplean principalmente en las ramas de comercio, actividades inmobiliarias y servicios empresariales y en la hostelería, y se identifica en ellas una proporción considerable de trabajadoras por cuenta propia. La trayectoria laboral seguida en España apunta a una reducida movilidad a nivel sectorial y ocupacional.

Un perfil laboral bastante alejado de los anteriores puede identificarse entre las mujeres procedentes de África, en su mayoría marroquíes. A este grupo nacional le son características unas bajas tasas de actividad y empleo, donde incluso variables como la edad o el nivel educativo apenas influyen. Las que están ocupadas lo hacen mayoritariamente en el comercio, la hostelería, las actividades inmobiliarias y de servicios empresariales y las actividades de los hogares, pero en este último caso en mucha menor proporción que para el resto de procedencias. Asimismo, se sitúan en los niveles inferiores de la escala laboral. Teniendo en cuenta esta distribución sectorial y ocupacional, y con base a sus niveles educativos -los más bajos en el conjunto de las distintas nacionalidades- podría hablarse de cierto ajuste entre el

fuentes estadísticas ofrecidas por el INE en su sistema público de información no permiten llegar a este nivel de desagregación de la Clasificación Nacional de Actividades Económicas (CNAE). 
perfil profesional y el empleo desempeñado. Son las más afectadas, en todo caso, por el desempleo. Su movilidad sectorial y ocupacional es de las más reducidas.

Para la nacionalidad más numerosa originaria del continente asiático, la china, se detecta la trayectoria laboral más lineal y homogénea. En el comercio y la hostelería se emplearían ocho de cada diez y a nivel ocupacional casi un $70 \%$ se ubica en la categoría de "trabajadoras de los servicios de restauración, personales, protección y vendedoras de comercio", presentando al mismo tiempo los índices más reducidos de movilidad sectorial y ocupacional (INE, 2007). Se trata del grupo nacional que en menor medida estaría afectado por el desempleo. Presentan, al mismo tiempo, una elevada presencia en el empleo por cuenta propia, donde es relevante la figura de "ayuda familiar".

Finalmente, otras procedencias con un peso relativo importante en España, Reino Unido y Alemania, mostrarían una importante falta de trayectorias laborales en nuestro país, con tasas de actividad y empleo bajas. Entre las ocupadas, Reino Unido registra una fuerte concentración en la rama de educación, seguida de lejos por el comercio, la hostelería o las actividades inmobiliarias y servicios empresariales. Las mujeres alemanas, por su parte, se distribuyen de forma más uniforme entre las ramas de actividad siendo, junto al comercio y la hostelería, la industria manufacturera la tercera rama en importancia de concentración.

Los tipos de ocupación que desempeñan se corresponderían con los de mayor nivel en la escala laboral, sobre todo entre las mujeres de Reino Unido, adecuándose a sus niveles educativos. Las condiciones laborales, entre ellas las salariales, son mejores incluso que para la media de las mujeres españolas. No registran altos índices de movilidad ocupacional, si bien hay que tener en cuenta que se sitúan en los niveles más altos de la escala laboral.

A modo de síntesis del estudio "Análisis de la situación laboral de las mujeres inmigrantes: modalidades de inserción, sectores de ocupación e iniciativas empresariales" (Sallé, Molpeceres y Ongil, 2009), la Tabla 1 resume las tendencias y matices comentados a lo largo de este apartado, en base al análisis del perfil, proyecto migratorio y situación laboral más común entre las procedencias mayoritarias de mujeres inmigrantes en España ${ }^{6}$.

\footnotetext{
${ }^{6}$ Tabla elaborada a partir de las distintas fuentes estadísticas utilizadas para el estudio: EPA, la Encuesta Nacional de Inmigrantes (ENI), los registros de afiliación a la Seguridad Social, Encuesta de Migraciones, Encuesta de Estructura Salarial y estadística de contratos registrados en el INEM. Refleja las situaciones observadas predominantes, sin posibilidad de señalar el dato concreto para cada indicador debido a la no comparabilidad de las fuentes, tanto por las variaciones de periodos de referencia en cada de una ellas, como por las distintas agrupaciones de regiones y países encontradas al referirse a población extranjera.
} 


\section{Tabla 1. Perfil, proyectos migratorios y situación laboral predominantes entre las mujeres extranjeras según países de procedencia mayoritarios}

\begin{tabular}{|c|c|c|c|}
\hline $\begin{array}{l}\text { PROCE- } \\
\text { DENCIA }\end{array}$ & PERFIL & $\begin{array}{c}\text { PROYECTO } \\
\text { MIGRATORIO } \\
\end{array}$ & SITUACIÓN LABORAL \\
\hline Rumania & $\begin{array}{l}\text { Etapa de } \\
\text { llegada a } \\
\text { España: a } \\
\text { partir de } 2002 \text {. } \\
\text { Nivel educati- } \\
\text { vo: secundaria } \\
\text { y superior. } \\
\text { Tasas de } \\
\text { fecundidad: } \\
\text { medias. } \\
\text { Estado civil: } \\
\text { casadas. } \\
\text { Edad media: } \\
32 \text { años. }\end{array}$ & $\begin{array}{l}\text { Motivos de traslado a } \\
\text { España: laborales (mejora } \\
\text { laboral) y por reagrupación } \\
\text { familiar. }\end{array}$ & $\begin{array}{l}\text { Nivel de actividad: alto. } \\
\text { Sectores de ocupación: actividades de los } \\
\text { hogares; actividades inmobiliarias y de } \\
\text { alquiler; servicios empresariales; comercio; } \\
\text { hostelería; y, en menor grado, industria } \\
\text { manufacturera. } \\
\text { Tipos de ocupación: trabajadoras no } \\
\text { cualificadas. } \\
\text { Autoempleo: relativamente alto }{ }^{7} \text {. } \\
\text { Trayectorias laborales: movilidad sectorial } \\
\text { media y ocupacional alta. }\end{array}$ \\
\hline Ecuador & $\begin{array}{l}\text { Etapa de } \\
\text { llegada a } \\
\text { España: } 1997- \\
2001 . \\
\text { Nivel educati- } \\
\text { vo: secundaria. } \\
\text { Tasas de } \\
\text { fecundidad: } \\
\text { altas. } \\
\text { Estado civil: } \\
\text { solteras, } \\
\text { casadas. } \\
\text { Edad media: } \\
31 \text { años. }\end{array}$ & $\begin{array}{l}\text { Motivos de traslado: } \\
\text { laborales (mejora de } \\
\text { empleo) y reagrupación } \\
\text { familiar. }\end{array}$ & $\begin{array}{l}\text { Nivel de actividad: muy alto. } \\
\text { Sectores de ocupación: actividades de los } \\
\text { hogares; hostelería; comercio; actividades } \\
\text { inmobiliarias y de alquiler, servicios } \\
\text { empresariales. } \\
\text { Tipos de ocupación: trabajadoras no } \\
\text { cualificadas; trabajadoras de servicios de } \\
\text { restauración, personales, protección y } \\
\text { vendedoras de los comercios. } \\
\text { Autoempleo: medio. } \\
\text { Trayectorias laborales: movilidad sectorial } \\
\text { y ocupacional alta. }\end{array}$ \\
\hline Marruecos & $\begin{array}{l}\text { Etapa de } \\
\text { llegada a } \\
\text { España: } 1997- \\
2001 . \\
\text { Nivel educati- } \\
\text { vo: sin } \\
\text { estudios, } \\
\text { primaria. } \\
\text { Tasas de } \\
\text { fecundidad: } \\
\text { muy altas. }\end{array}$ & $\begin{array}{l}\text { Motivos de traslado: } \\
\text { reagrupación familiar. }\end{array}$ & $\begin{array}{l}\text { Nivel de actividad: muy bajo. } \\
\text { Sectores de ocupación: comercio; hostele- } \\
\text { ría; actividades de los hogares; actividades } \\
\text { inmobiliarias y de alquiler; servicios } \\
\text { empresariales. } \\
\text { Tipos de ocupación: trabajadoras no } \\
\text { cualificadas; trabajadoras de servicios de } \\
\text { restauración, personales, protección; } \\
\text { vendedoras de los comercios; empleadas de } \\
\text { tipo administrativo. } \\
\text { Autoempleo: bajo. }\end{array}$ \\
\hline
\end{tabular}

${ }^{7}$ Posible efecto de la moratoria de libre circulación de personas trabajadoras para Rumania en España en el momento de la elaboración de esta tabla. 


\begin{tabular}{|c|c|c|c|}
\hline & $\begin{array}{l}\text { Estado civil: } \\
\text { casadas. } \\
\text { Edad media: } \\
25 \text { años. }\end{array}$ & & $\begin{array}{l}\text { Trayectorias laborales: movilidad sectorial } \\
\text { y ocupacional bajas. }\end{array}$ \\
\hline $\begin{array}{l}\text { Reino } \\
\text { Unido }\end{array}$ & $\begin{array}{l}\text { Etapa de } \\
\text { llegada a } \\
\text { España: } \\
\text { anterior al año } \\
2000 . \\
\text { Nivel educati- } \\
\text { vo: superior. } \\
\text { Tasas de } \\
\text { fecundidad: } \\
\text { bajas. } \\
\text { Estado civil: } \\
\text { casadas. } \\
\text { Edad media: } \\
51 \text { años. }\end{array}$ & $\begin{array}{l}\text { Motivos de traslado: } \\
\text { jubilación, laboral, } \\
\text { estudios. }\end{array}$ & $\begin{array}{l}\text { Nivel de actividad: bajo. } \\
\text { Sectores de ocupación: educación; } \\
\text { hostelería; actividades inmobiliarias y de } \\
\text { alquiler, servicios empresariales. } \\
\text { Tipos de ocupación: técnicas y profesiona- } \\
\text { les científicas e intelectuales; trabajadoras } \\
\text { de los servicios de restauración, personales, } \\
\text { protección y vendedoras de los comercios. } \\
\text { Autoempleo: bajo. } \\
\text { Trayectorias laborales: movilidad sectorial } \\
\text { media y ocupacional baja. }\end{array}$ \\
\hline Colombia & $\begin{array}{l}\text { Etapa de } \\
\text { llegada a } \\
\text { España: } 1997- \\
2001 . \\
\text { Nivel educati- } \\
\text { vo: secundaria. } \\
\text { Tasas de } \\
\text { fecundidad: } \\
\text { altas. } \\
\text { Estado civil: } \\
\text { solteras, } \\
\text { casadas. } \\
\text { Edad media: } \\
33 \text { años. }\end{array}$ & $\begin{array}{l}\text { Motivos de traslado: } \\
\text { laborales (mejora de } \\
\text { empleo) y reagrupación } \\
\text { familiar. }\end{array}$ & $\begin{array}{l}\text { Nivel de actividad: muy alto. } \\
\text { Sectores de ocupación: actividades de los } \\
\text { hogares; hostelería; comercio. } \\
\text { Tipos de ocupación: trabajadoras no } \\
\text { cualificadas; trabajadoras de servicios de } \\
\text { restauración, personales, protección y } \\
\text { vendedoras de los comercios. } \\
\text { Autoempleo: relativamente alto. } \\
\text { Trayectorias laborales: movilidad sectorial } \\
\text { y ocupacional altas. }\end{array}$ \\
\hline Bolivia & $\begin{array}{l}\text { Etapa de } \\
\text { llegada a } \\
\text { España: a } \\
\text { partir de } 2002 \text {. } \\
\text { Nivel educati- } \\
\text { vo: secundaria. } \\
\text { Tasas de } \\
\text { fecundidad: } \\
\text { altas. } \\
\text { Estado civil: } \\
\text { solteras, } \\
\text { casadas. } \\
\text { Edad media: } \\
32 \text { años. }\end{array}$ & $\begin{array}{l}\text { Motivos de traslado: } \\
\text { laborales (falta de empleo) } \\
\text { y reagrupación familiar. }\end{array}$ & $\begin{array}{l}\text { Nivel de actividad: muy alto. } \\
\text { Sectores de ocupación: actividades de los } \\
\text { hogares; hostelería; actividades inmobilia- } \\
\text { rias y de alquiler, servicios empresariales. } \\
\text { Tipos de ocupación: trabajadoras no } \\
\text { cualificadas; trabajadoras de servicios de } \\
\text { restauración, personales, protección y } \\
\text { vendedoras de los comercios. } \\
\text { Autoempleo: medio. } \\
\text { Trayectorias laborales: movilidad sectorial } \\
\text { y ocupacional bajas. }\end{array}$ \\
\hline
\end{tabular}




\begin{tabular}{|c|c|c|c|}
\hline Alemania & $\begin{array}{l}\text { Etapa de } \\
\text { llegada a } \\
\text { España: } \\
\text { anterior al año } \\
2000 \text {. } \\
\text { Nivel educati- } \\
\text { vo: superior. } \\
\text { Tasas de } \\
\text { fecundidad: } \\
\text { bajas. } \\
\text { Estado civil: } \\
\text { casadas. } \\
\text { Edad media: } \\
45 \text { años. }\end{array}$ & $\begin{array}{l}\text { Motivos de traslado: } \\
\text { jubilación, laboral, } \\
\text { estudios. }\end{array}$ & $\begin{array}{l}\text { Nivel de actividad: bajo. } \\
\text { Sectores de ocupación: comercio; activida- } \\
\text { des inmobiliarias y de alquiler, servicios } \\
\text { empresariales; industria manufacturera; } \\
\text { hostelería; transporte, almacenamiento y } \\
\text { comunicaciones. } \\
\text { Tipos de ocupación: técnicas y profesiona- } \\
\text { les científicas e intelectuales; trabajadoras } \\
\text { de servicios de restauración, personales, } \\
\text { protección y vendedoras de los comercios; } \\
\text { técnicas y profesionales de apoyo. } \\
\text { Autoempleo: bajo. } \\
\text { Trayectorias laborales: movilidad sectorial } \\
\text { baja y ocupacional media. }\end{array}$ \\
\hline Argentina & $\begin{array}{l}\text { Etapa de } \\
\text { llegada a } \\
\text { España: a } \\
\text { partir de } 2002 . \\
\text { Nivel educati- } \\
\text { vo: secundaria } \\
\text { y superior. } \\
\text { Tasas de } \\
\text { fecundidad: } \\
\text { medias-altas. } \\
\text { Estado civil: } \\
\text { casadas. } \\
\text { Edad media: } \\
36 \text { años. } \\
\end{array}$ & $\begin{array}{l}\text { Motivos de traslado: } \\
\text { reagrupación familiar, } \\
\text { laboral (mejora de em- } \\
\text { pleo). }\end{array}$ & $\begin{array}{l}\text { Nivel de actividad: alto. } \\
\text { Sectores de ocupación: comercio; activida- } \\
\text { des inmobiliarias y de alquiler, servicios } \\
\text { empresariales; industria manufacturera. } \\
\text { Tipos de ocupación: trabajadoras de } \\
\text { servicios de restauración, personales, } \\
\text { protección y vendedoras de los comercios; } \\
\text { técnicas y profesionales científicas e } \\
\text { intelectuales; técnicas y profesionales de } \\
\text { apoyo. } \\
\text { Autoempleo: relativamente alto. } \\
\text { Trayectorias laborales: movilidad sectorial } \\
\text { y ocupacional bajas. }\end{array}$ \\
\hline China & $\begin{array}{l}\text { Etapa de } \\
\text { llegada a } \\
\text { España: a } \\
\text { partir de } 2002 . \\
\text { Nivel educati- } \\
\text { vo: secundaria. } \\
\text { Tasas de } \\
\text { fecundidad: } \\
\text { bajas. } \\
\text { Estado civil: } \\
\text { casadas. } \\
\text { Edad media: } \\
29 \text { años. }\end{array}$ & $\begin{array}{l}\text { Motivos de traslado: } \\
\text { reagrupación familiar y } \\
\text { laboral (mejora de em- } \\
\text { pleo). }\end{array}$ & $\begin{array}{l}\text { Nivel de actividad: alto. } \\
\text { Sectores de ocupación: hostelería; comer- } \\
\text { cio. } \\
\text { Tipos de ocupación: trabajadoras de } \\
\text { servicios de restauración, personales, } \\
\text { protección y vendedoras de los comercios. } \\
\text { Autoempleo: bajo (aunque con predominio } \\
\text { de la figura de ayuda familiar). } \\
\text { Trayectorias laborales: movilidad sectorial } \\
\text { y ocupacional bajas. }\end{array}$ \\
\hline
\end{tabular}

Fuente: Sallé, Molpeceres y Ongil (2009: 241-244) 


\section{La procedencia, variable ilustrativa}

En paralelo al análisis descriptivo anterior se ha realizado un análisis de correspondencias múltiples tomando como fuente los microdatos de la $\mathrm{EPA}^{8}$.

Este análisis desvela las variables más significativas de la situación laboral de las mujeres extranjeras en España, así como los perfiles de mujeres predominantes conforme a ellas (Sallé, Molpeceres y Ongil, 2009).

De este análisis se desprende, como se mostrará a continuación, que la procedencia constituye la variable más ilustrativa respecto a la situación laboral de las mujeres extranjeras, resultando más explicativa que otras tales como la edad, el nivel educativo o la existencia de hijos o hijas menores en el domicilio. "Basta con conocer la procedencia de estas mujeres, para saber mucho sobre las realidades socioeconómicas que viven" (Sallé, Molpeceres y Ongil, 2009; 148). Otras variables relevantes son la edad o el tiempo de residencia en España.

La intención fue complementar el análisis descriptivo ya aportado con una visión sobre las distintas realidades de estas mujeres, identificables a través de agrupaciones con características socioeconómicas similares, sobre la base de operaciones estadísticas significantes. Cada agrupación obtenida se resume bajo un "perfil tipo" de mujer inmigrante, que muestra el conjunto de características socioeconómicas que más se repiten en la propia agrupación.

Para ello se ha construido lo que denominamos "Espacio socieconómico de las mujeres inmigrantes en España". Se trata de una representación gráfica en la que se reflejan qué rasgos socioeconómicos estructuran el total de la realidad socioeconómica de las mujeres inmigrantes en España, es decir, cuáles son los aspectos (variables) socioeconómicos clave, cuyo conocimiento ayuda a deducir todos los demás. En otras palabras, entre las modalidades de respuesta consideradas en el análisis, cuáles contienen la mayor cantidad de información respecto a todas las demás, permitiendo desentrañar las interrelaciones entre los aspectos socioeconómicos que estas mujeres presentan. Este tipo de análisis permite obtener perfiles más robustos, sintéticos y fiables que los aportados por el análisis estadístico descriptivo.

Se obtienen, así, tres ejes factoriales con los aspectos estructurantes del 'Espacio socioeconómico de las mujeres inmigrantes en España' (ver Gráfico 1), donde el orden de los ejes implica también un orden en la importancia de los rasgos socioeconómicos que los conforman como estructurantes de todos los demás.

${ }^{8}$ Gema de Cabo, Carolina Perondi y Covadonga Canteli han aplicado para su obtención técnicas estadísticas basadas en el mencionado análisis de correspondencias múltiples, tomando como fuente los microdatos de la EPA, con periodo de referencia de segundo trimestre de 2008 . 


\section{Gráfico 1. Espacio socioeconómico de las mujeres inmigrantes}

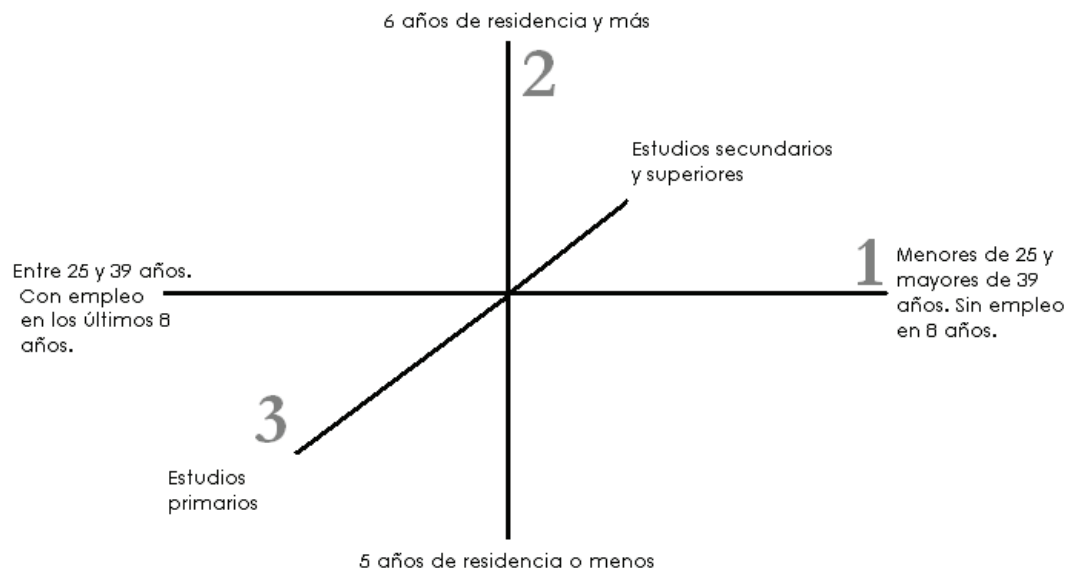

Fuente: "Análisis de la situación laboral de las mujeres inmigrantes: modalidades de inserción, sectores de ocupación e iniciativas empresariales" (Sallé, Molpeceres y Ongil, 2009)

- EJE 1: Estructurado por la actividad y la edad. El eje 1 opone a las que llevan al menos 8 años sin trabajar, que parecen tener o bien más de 40 años o bien menos de 25, en el lado positivo, con las demás: aquéllas que trabajan o lo hicieron hace menos de 8 años, y tienen entre 25 y 39 años. Se asocian a no tener ni haber tenido un empleo las siguientes nacionalidades: África, UE-27 (sin Rumania) y 'Otros' (es decir, EE.UU., Canadá, Japón, etc.). En el resto sí predominan las mujeres que en los últimos 8 años se han empleado.

- EJE 2: Estructurado por el tiempo de residencia. El eje 2 opone a las que llevan menos de 6 años residiendo en España, que proceden generalmente de Rumania y América Central y del Sur (sin incluir a Colombia ni a Ecuador), y no tienen hijos ni hijas menores a su cargo, situadas en el extremo negativo, a las que llevan 6 años o más residiendo en España. Estas últimas, en el lado positivo del eje, parecen tener hijos o hijas menores a su cargo y emplearse en sectores que requieren mayor formación. Proceden de África, Colombia, Ecuador y la Unión Europea (sin incluir Rumania).

- EJE 3: Estructurado por el nivel de formación. El eje 3 opone a las mujeres menos formadas, en el extremo negativo, con niveles primarios de estudios o sin estudios, y con menores a su cargo, a mujeres más formadas y característicamente sin hijos/hijas a su cargo, en el extremo positivo. Las primeras proceden de África, Colombia y Ecuador, las segundas son originarias en mayor medida de la Unión Europea, Rumania y América Latina.

De este primer análisis se extraen ya conclusiones relevantes para el objeto del estudio, como son: 
- La nacionalidad es una variable fuertemente determinante de la realidad socioeconómica de las mujeres inmigrantes. La nacionalidad es un aspecto muy relevante en la formación de cada uno de los tres ejes, lo que convierte esta dimensión en estructurante principal respecto al resto. Además, en cada eje, la nacionalidad viene acompañada de un segundo aspecto socioeconómico, lo que implica que está fuertemente correlacionada con todos los demás aspectos estructurantes: toda característica socioeconómica de las mujeres inmigrantes se ve fuertemente condicionada por su nacionalidad.

- Los hijos o hijas menores de 16 años en el domicilio no suponen una causa para alejarse del mercado laboral. En cambio, una procedencia determinada, o una edad muy joven o muy mayor, sí. La posición de la modalidad "con hijos o hijas menores de 16 años en domicilio" pone de manifiesto que la maternidad no es la causa de que haya mujeres inmigrantes que llevan al menos 8 años sin tener un empleo. Según el estudio de las correlaciones, la ausencia de empleo es un asunto condicionado más bien por la edad y el país de origen.

- El nivel de estudios queda relegado a condicionante de pequeño alcance a la hora de definir los perfiles, siendo más importante el tiempo de residencia en el país. En este caso, el nivel de estudios también parece estructurar el resto de características socioeconómicas, pero en menor nivel, en último lugar, por detrás incluso del tiempo de residencia. Esto quiere decir que el nivel de estudios tiene más variabilidad, sea cual sea la edad y sea cual sea el sector de actividad en que la mujer se emplea. En cambio, la importancia que muestra la variable "tiempo de residencia" podría reflejar la llegada por olas de mujeres inmigrantes de países determinados, olas que van satisfaciendo las necesidades del mercado laboral del momento, sin que el nivel de formación sea muy relevante.

Una vez construido el "Espacio Socioeconómico de las Mujeres Inmigrantes en España", con aportes significativos en sí mismo, se procede a realizar la clasificación o agrupación por perfiles, aplicando la técnica de clasificación en $k$-medias sobre las coordenadas principales obtenidas en el análisis de correspondencias múltiples.

Los aspectos que participaron en la determinación de los perfiles fueron los siguientes:

- Aspectos socioeconómicos, es decir, los anteriormente considerados del "Espacio socioeconómico": edad, nacionalidad, nivel de estudios, presencia de hijos o hijas menores de 16 años en el domicilio, tiempo de residencia en España, y sector de actividad en el que la persona se ve empleada?

${ }^{9}$ La construcción de esta última variable se realizó del siguiente modo:

- En caso de tener un empleo, se toma el sector de actividad del mismo.

- En caso de no tener un empleo, se asume el sector en el que se enmarcaba el último empleo que tuvo la persona, si éste fue hace menos de ocho años. 
Sobre estas variables la clasificación realizada da lugar a siete perfiles distintos, bastante equilibrados en el número mujeres que representan (todos ellos significaron entre el 11 y el $20 \%$ del total de la población de mujeres inmigrantes) y con un peso significativo -aunque no único ${ }^{10}$ - de determinadas nacionalidades en cada uno de ellos (según mostraba la construcción de su "Espacio Socioeconómico" acerca de la variable nacionalidad como aspecto estructurante).

- Aspectos laborales y de relación con el mercado de trabajo. Estas variables son estudiadas a posteriori, tomando como base los perfiles ya constituidos a partir de los rasgos socioeconómicos. Se tienen en cuenta las siguientes variables: relación con el mercado laboral, situación profesional, duración del contrato, segundo empleo, búsqueda de empleo, horas en el puesto de trabajo, tiempo en el empleo actual, ocupación ${ }^{11}$.

Tabla 2. Distribución por perfiles de las mujeres extranjeras en España (en edad de trabajar) (\%)

\begin{tabular}{|c|c|}
\hline Perfil 1 & $11 \%$ \\
\hline Perfil 2 & $20 \%$ \\
\hline Perfil 3 & $14 \%$ \\
\hline Perfil 4 & $14 \%$ \\
\hline Perfil 5 & $13 \%$ \\
\hline Perfil 6 & $13 \%$ \\
\hline Perfil 7 & $15 \%$ \\
\hline
\end{tabular}

Fuente: Sallé, Molpeceres y Ongil (2009:152)

- Si, por el contrario, hace más de 8 años que la persona encuestada no tiene un empleo o nunca lo ha tenido, entonces se distingue entre las mujeres que buscan un empleo y las mujeres que no lo buscan.

${ }^{10}$ Véase el caso de los perfiles 5 y 7 en los que, además de la nacionalidad (rumanas), resulta significativa para la conformación de perfiles diferenciados la variable edad.

${ }^{11}$ De forma similar a lo que ocurre con la variable sector de actividad, las variables "situación en el mercado laboral" y "ocupación" se calcularon a partir del empleo actual si la mujer encuestada estaba ocupada y, sobre el último empleo, si éste fue hace menos de 8 años y la mujer en cuestión se hallaba desempleada en el momento de la encuesta. Así, los porcentajes de modalidades de respuesta adoptadas se calcularon sobre el total de mujeres ocupadas en la actualidad o hace a lo sumo 8 años. Esto permitió ampliar las observaciones consideradas, es decir, las mujeres inmigrantes tenidas en cuenta, de un 59\% (las ocupadas) a un $73 \%$ (las ocupadas en los últimos 8 años). 
Los rasgos obtenidos para cada uno de estos perfiles fueron los siguientes:

\section{Perfil 1: colombianas, entre las más activas en el mercado laboral}

- Puede asociarse a la nacionalidad colombiana (el 65\% proceden de Colombia), aunque las ecuatorianas representan el 13\%.

- Son mayores que la media de mujeres inmigrantes, por cuanto la mitad de ellas tiene más de 40 años.

- Acumulan un tiempo considerable residiendo en España (un 76\% de ellas entre 7 y 10 años).

- Presentan diferencias respecto a la formación y sector de actividad en el que trabajan o han trabajado. Así, hay mujeres con estudios primarios o sin estudios (28\%), por un lado, y titulares de bachillerato (46\%), por otro.

- Las hay que se emplean o empleaban en los sectores de comercio (21\%), las actividades de los hogares (29\%), y también en los sectores agrupados bajo la rúbrica "Educación, sanidad, finanzas, telecomunicaciones..." (20\%).

- No tienen hijos ni hijas menores de 16 años viviendo en su domicilio (73\% de los casos).

- Se encuentran entre las más activamente integradas en el mercado laboral. Se trata del tercer perfil (tras el 6 y el 2) con menor porcentaje de "inactivas" (14\% frente al $29 \%$ de media).

- Se registra para este perfil un elevado porcentaje de mujeres ocupadas $(75 \%)$ y ocupadas subempleadas $(18 \%)$.

- Es el perfil que registra el porcentaje más elevado de mujeres que tienen un segundo empleo dentro de las ocupadas (10\%).

- Es característica la elevada presencia en ocupaciones no cualificadas (53\%).

- Asimismo, es llamativo el porcentaje de ellas que llevan 6 o más años trabajando en el mismo lugar (17\%).

- Tienen la segunda tasa de temporalidad más baja ( $40 \%$ de las asalariadas tienen contrato temporal).

Perfil 2: latinoamericanas, con el mayor porcentaje de ocupadas, concentrado en el sector de los hogares

- Provienen de países de América Central y del Sur en un 80\% (excluyendo a Ecuador y Colombia, que han sido considerados separadamente).

- Su media de edad está en los 34 años.

- Se trata de uno de los perfiles que menos tiempo lleva en España (el 88\% llegaron hace 5 años o menos).

- Son mujeres sin hijos o hijas menores de 16 años en el hogar (en un 62\% de los casos).

- Tienen un nivel educativo ligeramente por encima de la media (el 56\% de ellas tienen o bien el bachillerato o bien estudios superiores).

- Las define el hecho de estar empleadas, o haberlo estado, en el sector de actividades de los hogares (un 60\%). También es característico de este perfil el hecho de que el $18 \%$ se emplean, o empleaban, en los sectores agrupados bajo la rúbrica "Educación, sanidad, finanzas, telecomunicaciones...". 
- Al igual que ocurre con el perfil 1, se puede hablar de una mayor necesidad o un mayor deseo de estar empleadas: el perfil 2 reúne el mayor porcentaje de ocupadas $(79 \%)$ y de ocupadas subempleadas $(23 \%)$.

- Las mujeres ocupadas de este perfil destacan por llevar poco tiempo en su empleo actual (menos de un año, el $48 \%$ y entre 2 y 3 años, el $37 \%$ ).

- Con el mayor volumen de trabajadoras no cualificadas (el 61\%), las mujeres de este perfil probablemente se hallan entre las que acceden a condiciones laborales más precarias.

- Son las más proclives a buscar un segundo empleo (lo hace el $21 \%$ de las ocupadas). Su tasa de temporalidad, del $46 \%$, se sitúa un punto porcentual por encima de la media.

\section{Perfil 3: europeas, las de mayor edad y mayor nivel de formación}

- Son principalmente europeas (en un $75 \%$, sin incluir las mujeres rumanas) y procedentes de países agrupados bajo la rúbrica "otros" (EE.UU., China, Japón, en un $15 \%)$.

- Se trata de las de mayor edad (con una media de 44 años) y con más antigüedad en España (casi la mitad supera los 11 años de residencia).

- No tienen hijos o hijas menores de 16 años residiendo con ellas (un 65\%).

- Se caracterizan por su elevado grado de formación (el 50\% de ellas cursaron estudios superiores).

- Les distingue de la media, por un lado, que una alta proporción que emplea dentro de los sectores de "Educación, sanidad, finanzas, telecomunicaciones..." $(39 \%)$ y, por otro, un elevado porcentaje de mujeres que no se emplearon en España en los últimos 8 años y que no están buscando un empleo en la actualidad (31\%).

- Este perfil presenta un elevado porcentaje de mujeres "inactivas" (37\%), así como un elevado porcentaje de mujeres que no busca empleo dentro de las que no lo tienen $(84 \%)$.

- En cuanto las ocupaciones que desempeñan, destacan las técnicas y profesionales intelectuales $(37 \%)$ y las directoras de empresas privadas o Administraciones Públicas (9\%).

- Destaca este perfil por incluir al mayor porcentaje de empresarias con y sin personas asalariadas a su cargo (el 23\%).

- Se trata de mujeres con una antigüedad en sus puestos de trabajo superior la media $(33 \%$ frente a la media del $12 \%)$ y con menor tasa de temporalidad $(38 \%$ frente al $45 \%$ de media).

\section{Perfil 4: africanas, las menos formadas, las menos "activas"}

- El $81 \%$ de las mujeres que integran este perfil proceden de países africanos.

- Sus edades se concentran alrededor de la media, 34 años.

- Destacan por llevar mucho tiempo residiendo en España (9 años o más en el $46 \%$ de los casos).

- Se encuentran bajo este perfil las mujeres menos formadas: un $77 \%$ de ellas o bien cursaron estudios primarios o bien no cursaron estudios. 
- El porcentaje de madres con menores de 16 años en el domicilio dentro de este perfil es superior a la media de la población de mujeres inmigrantes (afecta al 67\%).

- 7 de cada 10 no ha trabajado en los últimos 8 años. Predomina, además, el hecho de que un $62 \%$ ni tiene ni busca un empleo en la actualidad.

- Presentan el más bajo porcentaje de mujeres ocupadas (20\%) y el porcentaje de "inactivas" es elevado (38\%).

- Las que sí tienen un empleo o lo han tenido en los últimos 8 años lo hacen principalmente en sectores como la agricultura (7\%) o la hostelería (9\%). En términos de ocupación lo hacen como trabajadoras no cualificadas (un 59\%) o en servicios de restauración y personales $(32 \%)$.

- Las mujeres que tienen un empleo en la actualidad lo tiene desde hace 6 y más años, aunque también son importantes los porcentajes de mujeres que llevan menos de 3 años en su empleo actual.

Perfil 5: rumanas de edades intermedias, con importantes tasas de ocupación

- Es característico de este perfil la elevada proporción de mujeres rumanas (35\%) con relación a la media.

- Se trata del segundo perfil más joven: el $89 \%$ tiene entre 25 y 39 años.

- En cuanto al tiempo de residencia, el 69\% de ellas llevan entre 4 y 6 años en España.

- Constituye, además, uno de los tres perfiles en los que el porcentaje de mujeres que convive con hijos o hijas menores de 16 años es más elevado (65\%).

- Comprende a mujeres muy formadas con relación al resto: el $42 \%$ ha cursado estudios superiores, el $26 \%$ posee el título de bachillerato y el $28 \%$ acredita secundaria. Es para este perfil que el porcentaje de mujeres sin estudios o con estudios primarios alcanza su valor mínimo (4\%).

- En cuanto a los principales sectores de actividad en los que se emplean o se han empleado, destacan la agricultura y la industria $(27 \%)$ y la hostelería $(26 \%)$.

- Este conjunto cuenta con un alto porcentaje de mujeres ocupadas $(65 \%)$ y de mujeres que tienen un segundo empleo (8\%).

- En cuanto al tiempo que llevan en el empleo actual, se puede decir que es algo inferior al de la población total, puesto que el porcentaje de mujeres que llevan más de 6 años en su puesto de trabajo actual es más bajo que en la media.

\section{Perfil 6: las madres de Ecuador, entre las más ocupadas}

- Perfil característicamente ecuatoriano (con un $67 \%$ de mujeres procedentes de dicho país).

- Al igual que en el caso del perfil anterior, el perfil 6 contiene a mujeres de edades centrales (el $85 \%$ de estas mujeres tienen entre 25 y 39 años).

- También presentan un alto grado de concentración en cuanto a los años de residencia en España: el 93\% llevan en el país entre 6 y 10 años.

- Puede ser calificado como el de "las madres", por presentar el más alto porcentaje de mujeres que tienen en su domicilio hijos o hijas menores de 16 años: $81 \%$.

- Se trata también del perfil con la más alta proporción de mujeres que poseen el bachillerato (50\%). El título de secundaria también es característico $(26 \%)$. 
- En cuanto a los sectores de actividad predominantes, se trata de la agricultura y la industria (21\%), el comercio (19\%) y la hostelería (25\%).

- Presenta el segundo mayor porcentaje de ocupadas (78\%), después del perfil 2, lo mismo que ocurre para el porcentaje de ocupadas subempleadas (20\%). Además, reúne el menor porcentaje de "inactivas" $(10 \%)$.

- En cuanto a sus ocupaciones actuales o las de su último empleo, merecen ser destacados los porcentajes de trabajadoras cualificadas y de empleadas de tipo administrativo (9\% y $8 \%$, respectivamente). El porcentaje de trabajadoras en restauración y servicios personales ronda el $33 \%$ y el de trabajadoras no cualificadas el $45 \%$.

- En cuanto al tiempo que llevan en su empleo actual, la distribución es muy similar a la de la población total de mujeres inmigrantes, con un $75 \%$ que acumula 3 años o menos.

\section{Perfil 7: las rumanas más jóvenes, las que menos tiempo llevan en España}

- Al igual que ocurría en el caso del perfil 5, las mujeres procedentes de Rumania se ven aquí significativamente representadas (son el $48 \%$ de las clasificadas). La diferencia entre ambos perfiles es que en éste se hallan las mujeres rumanas más jóvenes, teniendo un $70 \%$ de ellas entre 16 y 24 años. Su juventud determina, sin duda, el resto de características de este perfil.

- Llevan menos tiempo en España (el 32\% con 2 años o menos), su nivel de estudios es inferior (el porcentaje de mujeres con estudios superiores se queda en 5\%, mientras que ascendía a $42 \%$ en el perfil 5) y no tienen hijos ni hijas menores de 16 años en su domicilio (sólo el 12\%).

- Además, ni tienen ni han tenido un empleo en los últimos 8 años en una elevada proporción $(47 \%)$.

- Aquellas mujeres del perfil 7 que sí trabajan, lo hacen en el sector de hostelería (un $25 \%$ ).

- Las características laborales de las que trabajan también parecen desprenderse de la joven edad de sus integrantes. Un ejemplo de ello es el alto porcentaje de mujeres paradas que buscan un primer empleo dentro de este perfil.

- Además, se registra el segundo porcentaje de "inactivas" más elevado (42\%, detrás del perfil 2).

- Se caracterizan por llevar poco tiempo en su empleo (menos de un año, el 61\% de las ocupadas).

- Asimismo, sufren la más elevada tasa de temporalidad, con un 53\% de contratos temporales.

- Más de la mitad de estas mujeres, si tienen o han tenido un empleo, lo han hecho como trabajadoras en servicios de restauración y otros servicios personales.

\section{Conclusiones}

Tras el análisis presentado podemos concluir que, efectivamente, la situación laboral generalizada de las mujeres inmigrantes en España viene marcada por condi- 
ciones precarias -en términos de tipo de ocupación, sobrecualificación, inestabilidad, condiciones laborales, etc.- pero teniendo en cuenta que ello está condicionado y matizado por variables como la edad, el tiempo de residencia en España y, fundamentalmente, por la nacionalidad.

La variable país de procedencia/nacionalidad es la que guarda una mayor relación respecto a la situación laboral, incluso por encima de otras como pueden ser la edad, el nivel educativo, o las responsabilidades familiares dentro del hogar.

El mercado laboral mostraría, de esta forma, una marcada estratificación laboral en función del género (hombre/mujer), el origen (autóctono/extranjero) y el estatus migratorio (cómo y de dónde se procede) en los países receptores de migración, situando a estas mujeres en una posición de "infra-clase" laboral y desarrollando trabajos en los nichos laborales menos deseables en cuanto a remuneración, condiciones laborales, protecciones legales y reconocimiento social. De manera que no se trata sólo de que sean mujeres las que realicen ciertos trabajos en función de sus roles de género, sino que los desempeñan mujeres de determinada raza, clase, origen étnico y/o nacionalidad (Pérez, Paiewonsky y García, 2008). Las dimensiones de género, clase social, etnicidad o grupo nacional se entrecruzan, abocando a las mujeres inmigrantes a una participación laboral máximamente precaria y marginal.

Dicha situación de empleo reclama ser visibilizada y evidenciada desde la labor de investigación que nos ocupa. Ahora bien, igualmente es cierto que junto al reconocimiento de la precariedad y la discriminación que muchas de estas mujeres padecen es preciso hacer balance sobre los efectos positivos que la migración laboral aporta. De hecho, a la denuncia y reivindicación conviene sumar una visión sobre el nuevo e importante papel de las mujeres inmigrantes en las sociedades de origen y de destino, como reconocimiento a estas mujeres, pero también como mecanismo que pudiera repercutir en su empoderamiento individual y colectivo (como trabajadoras, emprendedoras y ciudadanas), dado que, todavía hoy, una parte muy sustantiva de esta historia está pendiente de escribir y queda por delante mucha tarea por hacer.

\section{Bibliografía}

Cáceres, J.I., Escot L., Fernández J.A, Saiz, J. (2004). La segregación ocupacional $y$ sectorial de la mujer en el mercado de trabajo español. Documentos de trabajo de la Facultad de Ciencias Económicas y Empresariales. Universidad Complutense de Madrid.

Cachón, L. (2004). Diez notas sobre la inmigración en España y una conclusión ni inesperada ni desesperada. Tribuna Americana 4. La inmigración en España, más allá del tópico. Casa de América. 
Camacho, G., Hernández, K. (2005). Cambió mi vida: migración femenina, percepciones e impactos. UNIFEM.

Camacho, G. (2010). Mujeres inmigrantes. Trayectoria laboral y perspectiva de desarrollo humano. Colección Cátedra Iberoamericana de estudios sobre migraciones. CLACSO.

COLECTIVO IOE. (1998). Mujeres inmigrantes en España. Proyectos migratorios $y$ trayectorias de género. OFRIM, Suplementos.

ENI. (2007). Encuesta Nacional de Inmigrantes. INE.

Gregorio, C. (1997). El estudio de las migraciones internacionales desde una perspectiva de género. Instituto Universitario de Estudios sobre Migraciones.

González, A. (2008). Estrategias familiares y laborales en la emigración. Reagrupación familiar, elección de parejas y empleo de los inmigrantes en el país de destino. Colección de Estudios. CES.

Ibáñez, M. (2008). La segregación ocupacional por sexo a examen. Características personales, de los puestos y de las empresas asociadas a las ocupaciones masculinas y femeninas. Revista Española de Investigaciones Sociológicas (Reis) número 123.

INE (2010). Encuesta de Población Activa. Resultados anuales.

INE (2010). Padrón municipal: explotación estadística y Nomenclátor. Explotación estadística del Padrón. Principales series de población desde 1998. 2000-2010.

INE (2011). Encuesta de Población Activa. IIT 2011.

Jolly, S. y Reeves, H. (2005). Gender and migration. Overview Report. Bridge.

Martínez, J. (2007). Feminización de las migraciones en América latina: discusiones y significados para políticas. Taller sobre Programas para Trabajadores Migratorios Temporales. El Salvador.

Maté, J.J., Nava, L.A., Rodríguez, J.C. (2002). La segregación ocupacional por razón de sexo en la economía española, 1994-1999. Revista del Ministerio de Trabajo y Asuntos Sociales. Número 36, Economía y Sociología. MTIN.

Morrison, A.R; Schiff, M., Sjöblom, M. (2007). The international migration of women. Banco Mundial.

ONU (2009). International Migration Report 2006: A Global Assessment. 
Orozco, A., Paiewonsky, D. y García, M. (2008). Cruzando Fronteras II: Migración y desarrollo desde una perspectiva de género. INSTRAW.

Orozco, A. (2007). Cadenas globales de cuidado. Género, Remesas y Desarrollo. Documento de Trabajo 2. United Nations INSTRAW.

Oso, L. (2004). El empresariado étnico como una estrategia de movilidad social para las mujeres inmigrantes. Instituto de la Mujer.

Oso, L. (2008). Migración, género y hogares transnacionales. En GARCIA, J. y LACOMBA, J. "La inmigración en la sociedad española: una radiografía multidisciplinar". Ediciones Bellaterra S.A.

Parella, S. (2003) Mujer, inmigrante y trabajadora: la triple discriminación. Barcelona: Ed. Anthropos.

Paiewonsky, D. (2008). Impactos de las migraciones en la organización social de los cuidados en los países de origen: el caso de República Dominicana. Ponencia presentada en el curso Mujeres que migran, mujeres que cuidan: la nueva división sexual del trabajo, 1, 2 y 3 de diciembre de 2008, Madrid. UN-INSTRAW y ACSUR Las Segovias.

Pérez, A.; Paiewonsky, D.; García, M. (2008). Cruzando Fronteras II: Migración y desarrollo desde una perspectiva de género. INSTRAW.

Sallé, MA., Molpeceres, L., Óngil, M. (2009). Análisis de la situación laboral de las mujeres inmigrantes: modalidades de inserción, sectores de ocupación e iniciativas empresariales. Colección Estudios $\mathrm{N}^{\mathrm{o}} 110$. Instituto de la Mujer.

Solé, C.; Parella, S. y Cavalcanti, L. (2007). El empresariado inmigrante en España. Fundación La Caixa.

Sorensen, N. (2005). Migración, género y desarrollo: el caso dominicano. En Zúñiga, N. (ed.) "La migración: un camino entre el desarrollo y la cooperación". Madrid, CIP-FUHEM.

UNITED NATIONS, DEPARTMENT OF ECONOMIC AND SOCIAL AFFAIRS, POPULATION DIVISION (2009). International Migration Report 2006: A Global Assessment. 